\title{
American Board of Family Medicine (ABFM) Announces Further Enhancements to Maintenance of Certification for Family Physicians (MC-FP)
}

The beginning of 2010 officially completed the staged, 7-year transition of every single American Board of Family Medicine (ABFM) Diplomate into the new recertification paradigm: Maintenance of Certification for Family Physicians (MC-FP). At its inception in 2003, the MC-FP was designed to assist with the task of providing high-quality care to patients while at the same time fitting into physicians' busy day-to-day professional lives. In keeping with these 2 basic design principles, during the transition the ABFM listened carefully to feedback and concerns and continuously improved MC-FP to simplify and streamline the process, target it to practice needs, and create flexibility in how MC-FP requirements were met.

The MC-FP has changed dynamically over time. The ABFM has systematically surveyed the medical literature and, as new information that would potentially change the quality of care delivered to patients became available, the ABFM incorporated that information into the continually updated self-assessment modules. As new methods of measurement have been validated, they have been incorporated into the examination processes to more accurately assess the physician's knowledge base. As new tools became available for assessing and evaluating patients, clinical simulation technology has been modified to take advantage of these advances. The ABFM promised to remain on the cutting edge of physician assessment and has worked diligently to keep that promise.

Penetration of MC-FP has been relatively uniform across the United States and has not been constrained by geography, practice setting, or physician demographics. Despite a rocky first year after the rollout of MC-FP, uptake has been consistent and has conformed to the ABFM's original assumptions about participation. This has been in large part because of the excellent suggestions that Diplomates have offered to improve the process along the way. Although there were many useful suggestions during the transition, 2 important messages stood out. First, the examination need not be given as frequently as in the past, given the multiple ways in which the ABFM now assesses Diplomates' competencies; by overwhelmingly choosing the 10-year option over the 7-year MC-FP pathway during the transition, almost all Diplomates passionately voted on this issue. Secondly, although flexibility of when and how MC-FP requirements were completed has been greatly enhanced, Diplomates have asked for even more options.

The Diplomates have spoken, and the ABFM has listened. The ABFM is happy to announce some important enhancements to MC-FP that will be introduced starting in 2011. Beginning with those who certify or recertify next summer, the examination will be temporally unlinked from the MC-FP cycle. Currently the MC-FP culminates with the administration of the examination at the end of the cycle, but with this change it may be taken at any point in time during the MC-FP cycle, and the results will be valid for 10 years. In addition, Diplomates will be allowed to choose how to complete their part II (self-assessment modules) and part IV (performance in practice modules, methods in medicine modules, or approved alternatives) requirements during each 3-year stage. Each module will be assigned a point value, and each Diplomate will need to accumulate a sufficient number of points every 3 years (the exact total number of points is yet to be determined). How this is accomplished is up to the Diplomate as long as the combination of completed activity includes at least one part II module and one part IV module.

Diplomates must continuously meet the requirements of all 4 parts of the MC-FP to remain certified. With this new format in place, each Diplomate will simply need to meet 4 requirements 
during each 3-year stage of the MC-FP cycle to maintain certification:

1. Meet ABFM licensure requirements (part I).

2. Complete a part II and part IV activity totaling a set number of points in any combination of the Diplomate's choosing (Diplomates will continue to receive continuing medical education credits for this activity).

3. Meet ABFM continuing medical education requirements (part II).

4. Perform successfully on the examination within the last 10 years (part III).

The ABFM believes that these new changes will also allow it to begin issuing certificates without end dates, beginning in the summer of 2011. The validity of these newly issued certificates would be continuous as long as the first 3 above-listed re- quirements had been met by the end of each 3-year stage in MC-FP and the Diplomate had performed successfully on the examination within the previous 10 years. Accordingly, last month the ABFM submitted a request to the American Board of Medical Specialties Committee on Certification seeking permission to do this. The American Board of Pediatrics began issuing certificates without end dates at the beginning of 2010, and the American Board of Internal Medicine soon will make a similar request to also begin in 2011. That all 3 primary care boards have contemplated these changes at the same time is not coincidence. The ABFM, the American Board of Pediatrics, and the American Board of Internal Medicine have been working together diligently for the past 5 years in an effort to harmonize the MOC programs of each board.

James C. Puffer, MD American Board of Family Medicine 